\title{
Validating Streaming XML Documents
}

\author{
Luc Segoufin \\ INRIA-Rocquencourt \\ Luc.Segoufin@inria.fr
}

\author{
Victor Vianu* \\ U.C. San Diego \\ vianu@cs.ucsd.edu
}

\begin{abstract}
This paper investigates the on-line validation of streaming XML documents with respect to a DTD, under memory constraints. We first consider validation using constant memory, formalized by a finite-state automaton (FSA). We examine two flavors of the problem, depending on whether or not the XML document is assumed to be well-formed. The main results of the paper provide conditions on the DTDs under which validation of either flavor can be done using an FSA. For DTDs that cannot be validated by an FSA, we investigate two alternatives. The first relaxes the constant memory requirement by allowing a stack bounded in the depth of the XML document, while maintaining the deterministic, one-pass requirement. The second approach consists in refining the DTD to provide additional information that allows validation by an FSA.
\end{abstract}

\section{INTRODUCTION}

The Extended Markup Language (XML) is emerging as the standard for data exchange on the Web. Many applications, ranging from e-commerce and B2B to scientific applications monitoring sensor or satellite data, increasingly require on-line processing of large amounts of data in XML format using limited memory. Such processing includes querying XML documents, computing running aggregates of streams of numerical data, and validating XML documents against given Document Type Definitions (DTDs).

In this paper we take a first step towards a formal investigation of processing streaming XML documents, by studying the validation question. This is an important practical problem, which is already being tackled in in-

\footnotetext{
${ }^{*}$ This author supported in part by the NSF under grant number IIS-9802288.
}

dustry, with some commercial products developed (see related work below).

In its most restrictive form, the problem of validating streaming XML is to verify that an XML document is valid with respect to a given DTD in a single pass and using a fixed amount of memory, depending on the DTD but not on the size of the XML document. In other words, validation is done by a finite-state automaton (FSA) performing a pass on the XML document as it streams through the network, with constant memory. The problem comes in two flavors, depending on whether or not validation includes checking that the input is a well-formed XML document. Validation that includes checking well-formedness is referred to as strong validation. Checking satisfaction of the DTD under the assumption that the input is a well-formed XML document is referred to simply as validation. It is easy to see that validation of either flavor is not possible for all DTDs using an FSA. DTDs for which (strong) validation can be done using an FSA are referred to as (strongly) recognizable DTDs.

The main results of the paper provide conditions on DTDs under which they are (strongly) recognizable. The characterization of strongly recognizable DTDs is straightforward: the DTD has to be non-recursive. Characterizing recognizable DTDs is much more intricate and technically difficult. To put the problem in perspective, note that validation with respect to a DTD amounts to checking membership of the tree associated with the XML document in a regular tree language, while validation by an FSA amounts to acceptance of the tree by a restricted form of tree-walking automaton. Thus, the connection between FSA and DTDs can be viewed as a variant (albeit simpler) of the connection between tree-walking automata and regular tree languages, a long-standing open problem [8, 9]. We obtain several kinds of results. First, we precisely characterize recognizable DTDs when the DTDs are "fully recursive", i.e. all element tags that lead to recursive tags are mutually recursive. The condition we provide can be tested in ExPTIME with respect to the size of the DTD, and in polynomial time for DTDs using 1unambiguous regular expressions, as required by XMLSchema [4]. As a side effect, we obtain an algorithm for 
constructing from a fully recursive DTD a standard FSA that (i) always accepts only documents valid w.r.t. the DTD (but possibly more), and (ii) accepts precisely the documents valid w.r.t. the DTD, whenever the DTD is recognizable. The standard FSA can be constructed in time exponential in the DTD.

For DTDs that are not fully recursive, a precise characterization of recognizability remains an open question. We provide a set of necessary conditions for recognizability, as well as an extension yielding a sufficient condition. Furthermore, the construction of the standard FSA can be extended from the fully recursive case to arbitrary DTDs. It turns out that the sufficient condition is a characterization of the DTDs for which the standard FSA accepts precisely the documents valid with respect to the DTD.

For the case when validation using an FSA is not possible, we consider several alternatives. First, we relax the constant memory requirement and allow as auxiliary memory a stack of depth linear in the depth of the XML document. This is often reasonable in practice, since XML documents are typically fairly shallow, although they may be very large. We show that every DTD can be validated by a deterministic pushdown automaton whose stack is linear in the depth of the input document. Moreover, this holds even for DTDs extended with specialization, a form of element subtyping present in recent proposals such as XML-Schema. An orthogonal approach is to explore whether non-recognizable DTDs can be tweaked in reasonable ways so as to become recognizable. We show that for every DTD one can find a specialization of it which is recognizable. Intuitively, this is obtained by refining the tags of the original DTD to include more information useful for quick validation. This provides a trade-off between "accuracy" of the tags and the ability to perform efficient streaming validation.

Although limited to validation, this paper provides necessary groundwork for further investigating the problem of querying streaming XML documents. Indeed, the technical machinery developed here is likely to be useful for the more complex querying problem.

\section{Related work}

As far as we know, there is no formal work on validating or querying streaming XML. Heuristics for the evaluation of regular path queries in streaming XML documents are considered in [12]. This is part of a larger prototype, called Tukwila, designed for processing streaming XML documents currently developed in the University of Washington [13].

The Streaming XML Validator is a commercial product from TIBCO that performs validation of streaming XML with respect to a DTD (see http://www.tibco.com). To our understanding, their approach is based on traditional parsing techniques enhanced with heuristics geared towards streaming inputs.
A lot of work has been done on continuous queries over the Internet [6, 3] and on query subscription [16, 14]. In this scenario the query is fixed and outputs a stream of data produced on-line from an incoming stream of data. The emphasis is on filtering and incremental maintenance of views, including aggregate functions. Another large body of work focuses on numerical data streams such as sensor data.

The paper is organized as follows. Our abstraction of XML documents and DTDs, as well as basic notions on tree automata are reviewed in the Preliminaries. Section 3 concerns strongly recognizable DTDs. Section 4 presents the results on recognizable DTDs. Alternative approaches to validation are described in Section 5. Finally, brief conclusions are provided in Section 6 .

\section{PRELIMINARIES}

We introduce here the basic formalism used throughout the paper, including our abstraction of XML documents and DTDs. We also recall informally some basic notions related to tree automata and languages.

Let $\Sigma$ be a finite alphabet.

\section{Tree document}

We abstract XML documents by "tree documents" capturing the nesting structure of elements in the document. A tree document $t$ over $\Sigma$ is a finite unranked tree with labels in $\Sigma$ and an order on the children of each node. The following represents a simple tree document.

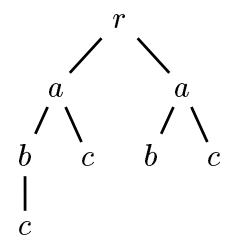

\section{String representation}

XML documents are a string representation of trees using opening and closing tags for each element. A streaming XML processor sees the sequence of opening and closing tags in the order in which they appear in the document. It is therefore useful to consider explicitly this string representation of an XML document. For each $a \in \Sigma$ let $a$ itself represent the opening tag and $\bar{a}$ represent the closing tag for $a$. Let $\bar{\Sigma}=\{\bar{a} \mid a \in \Sigma\}$. With this notation, the string associated to the tree document above is $r a b c \bar{c} \bar{b} c \bar{c} \bar{a} a b \bar{b} c \bar{c} \bar{a} \bar{r}$. More generally, we associate to each tree document $t$ a string representation denoted $[t]$ and defined inductively as follows: if $t$ is a single root labeled $a$, then $[t]=a \bar{a}$; if $t$ consists of a root labeled $a$ and subtrees $t_{1} \ldots t_{k}$ then $[t]=a\left[t_{1}\right] \ldots\left[t_{k}\right] \bar{a}$. Note that $\Sigma$ and $\bar{\Sigma}$ can be viewed as opening and closing multisorted parenthesis, and for each tree document $t$ the string $[t]$ is a well-balanced string over $\Sigma \cup \bar{\Sigma}$ corresponding to a depth-first traversal of $t$. If $T$ is a set of tree 
documents, we denote by $\mathcal{L}(T)$ the language consisting of the string representations of the tree documents in $T$.

\section{Tree types and DTDs}

DTDs and their variants provide a typing mechanism for XML documents. We will use several notions of types for trees. The first corresponds closely to the DTDs proposed for XML documents, and we therefore (by slight abuse) continue to use the same term. A DTD consists of an extended context-free grammar ${ }^{1}$ over alphabet $\Sigma$ (we make no distinction between terminal and non-terminal symbols). A tree document over $\Sigma$ satisfies a DTD $d$ (or is valid w.r.t. $d$ ) if it is a derivation tree of the grammar. For example, the tree document $\begin{aligned} & r \rightarrow a^{*} \\ \text { above is valid w.r.t. the } \mathrm{DTD}^{2}: & a \rightarrow b c \\ & b \rightarrow c^{?} \\ & c \rightarrow \epsilon\end{aligned}$ lar expressions are closed under union, we can assume w.l.o.g. that each DTD has a unique rule $a \rightarrow R_{a}$ for each symbol $a \in \Sigma$. In the following $R_{a}$ will denote both the regular expression and the corresponding regular language. The set of tree documents satisfying a DTD $d$ is denoted by $S A T(d)$. We also denote by $\mathcal{L}(d)$ the language over $\Sigma \cup \bar{\Sigma}$ consisting of the string representations of all tree documents in $S A T(d)$, that is $\{[t] \mid t \in S A T(d)\}$. Clearly, $\mathcal{L}(d)$ is a context-free language for every DTD $d$. In fact, such languages of wellbalanced strings of multisorted parenthesis have been studied in formal language theory under the name of Dyck languages [10].

The most recent DTD proposal, called XML-Schema, imposes a restriction on the regular expressions associated with each symbol: the expressions have to be 1-unambiguous. This property guarantees that the deterministic FSA for the regular expression is polynomial in the expression. Such regular expressions and other variants are studied formally in [4].

We next consider an extension of basic DTDs, also present in XML-Schema. This is motivated by a severe limitation of DTDs: their definition of the type of a given tag depends only on the tag itself and not on the context in which it occurs. For example, this means that the singleton tree document represented above cannot be described by a DTD, because the "type" of the first $b$ differs from that of the second $b$. This naturally leads to an extension of DTDs with specialization (also called decoupled types) which, intuitively, allows defining the type of a tag by several "cases" depending on the context. Specialized DTDs have been studied in [17] and are equivalent to formalisms proposed in [2, 7]. They are present in a restricted form in XML-Schema.

Formally, we have:

\footnotetext{
${ }^{1}$ In an extended $\mathrm{CFG}$, the right-hand sides of productions are regular expressions over the terminals and nonterminals.

${ }^{2} c^{?}$ is an abbreviation for $(c \mid \epsilon)$.
}

Definition 2.1. A specialized DTD over $\Sigma$ is a tuple $d=\left(\Sigma, \Sigma^{\prime}, d^{\prime}, \mu\right)$ where

$$
\begin{aligned}
& \text { - } \Sigma \text { and } \Sigma^{\prime} \text { are finite alphabets; } \\
& \text { - } d^{\prime} \text { is a DTD over } \Sigma^{\prime} \text {; and } \\
& \text { - } \mu \text { is a mapping from } \Sigma^{\prime} \text { to } \Sigma \text {. }
\end{aligned}
$$

A tree document $t$ over $\Sigma$ satisfies a specialized DTD $d$, if $t \in \mu\left(S A T\left(d^{\prime}\right)\right)$.

Intuitively, $\Sigma^{\prime}$ provides for some $a$ 's in $\Sigma$ a set of specializations of $a$, namely those $a^{\prime} \in \Sigma^{\prime}$ for which $\mu\left(a^{\prime}\right)=a$. We also denote by $\mu$ the homomorphism induced on strings and trees by $\mu$, extended whenever needed to symbols in $\bar{\Sigma}^{\prime}$ by $\mu\left(\bar{a}^{\prime}\right)=\overline{\mu\left(a^{\prime}\right)}$.

\section{Tree automata}

We assume familiarity with basic notions of language theory, including (nondeterministic) finite-state automata ( (N)FSA), context-free grammar (CFG) and language (CFL), and (deterministic) push-down automaton ((D)PDA) (e.g., see [11]).

We also use results on regular tree languages and tree automata. Regular tree languages are natural extensions to trees of the familiar string regular languages, and are classically defined for binary trees. A nondeterministic top-down regular tree automaton over $\Sigma$ has a finite set $Q$ of states, including a distinguished initial state $q_{0}$ and an accepting state $q_{f}$. In a computation, the automaton labels the nodes of the tree with states, according to a set of rules, called transitions. An internal node transition is of the form $(a, q) \rightarrow\left(q^{\prime}, q^{\prime \prime}\right)$, for $a \in \Sigma$. It says that, if an internal node has symbol $a$ and is labeled by state $q$, then its left and right children may be labeled by $q^{\prime}$ and $q^{\prime \prime}$, respectively. A leaf transition is of the form $(a, q) \rightarrow q_{f}$ for $a \in \Sigma$. It allows changing the label of a leaf with symbol $a$ from $q$ to the accepting state $q_{f}$. Each computation starts by labeling the root with the start state $q_{0}$, and proceeds by labeling the nodes of the trees non-deterministically according to the transitions. The input tree is accepted if some computation results in labeling all leaves by $q_{f}$. A set of complete binary trees is regular iff it is accepted by some top-down tree automaton. Regular languages of finite binary trees are surveyed in [18]. The extension to the unranked case is discussed in [5]. Regular tree languages have similar closure properties to regular string languages, in both the ranked and unranked cases. It is worth noting that regular tree languages can be defined by many other equivalent formalisms, including bottom-up (non)deterministic automata and Monadic Second-Order logic (MSO) on the standard structures associated to trees. Interestingly, it turns out that specialized DTDs are precisely equivalent to top-down nondeterministic tree automata over unranked trees [5, 17]. Thus, they define precisely the regular tree languages. 
This is more evidence that specialized DTDs are a robust and natural specification mechanism.

Another useful kind of automata on trees are the treewalking automata (defined by [1] for the ranked case). These are more sequential in nature than the automata described earlier: there is a head that resides at any time at a single given node. In the unranked version, transitions depend on the current label and the state, and consist of moving the head up, down (on the leftmost child), or horizontally to the left or right neighbor. It is easily seen that trees accepted by tree-walking automata can be defined in MSO, so are regular tree languages. Conversely, it is conjectured that tree-walking automata can only define a strict subset of the regular tree languages $[8,9]$.

\section{STRONG VALIDATION OF XML DOC- UMENTS}

We begin with the strong validation problem for streaming tree documents. Recall that checking well-formedness of the XML document is now part of the validation problem. More formally, let $d$ be a DTD (possibly specialized) over $\Sigma$ and consider the associated string language $\mathcal{L}(d)$ over $\Sigma \cup \bar{\Sigma}$. We wish to characterize the DTDs $d$ for which $\mathcal{L}(d)$ can be recognized by an FSA, i.e. $\mathcal{L}(d)$ is regular. Such DTDs are called strongly recognizable. We first illustrate the problem with two examples.

ExAMPLE 3.1. : Consider the DTD $d: \begin{aligned} & r \rightarrow a \\ & a \rightarrow a\end{aligned}$ ?

which defines the trees with root $r$ containing a single branch of arbitrary length of nodes labeled $a$. Thus, $\mathcal{L}(d)=\left\{r a^{n} \bar{a}^{n} \bar{r} \mid n \in \mathbb{N}\right\}$ which is not regular. So, $d$ cannot be strongly validated by an FSA and is not strongly recognizable.

EXAMPLE 3.2. : Consider the DTD $d: \begin{aligned} & r \rightarrow a^{*} \\ & a \rightarrow b \mid c\end{aligned}$ Now $\mathcal{L}(d)=r(a(b \bar{b} \mid c \bar{c}) \bar{a})^{*} \bar{r}$ which is regular. So, $d$ is strongly recognizable.

We provide a complete characterization of the strongly recognizable (specialized) DTDs: they are precisely the non-recursive ones, defined next together with other related notions used throughout the paper.

Definition 3.1. Let d be a DTD over $\Sigma$ and $G_{d}$ the graph constructed as follows: its set of vertices is $\Sigma$, and for each rule $a \rightarrow R_{a}$ in $d$ there is an edge from $a$ to $b$ for each $b$ occurring in some word in $R_{a}$. We call $G_{d}$ the dependency graph of $d$. Two labels $a$ and $b$ are mutually recursive if they belong to some cycle of $G_{d}$, and $a$ is recursive if it is mutually recursive with itself. The DTD $d$ is non-recursive iff $G_{d}$ is acyclic. Similarly, a specialized $D T D d=\left(\Sigma, \Sigma^{\prime}, d^{\prime}, \mu\right)$ is non-recursive iff the DTD d' over $\Sigma^{\prime}$ is non-recursive. Finally, a DTD $d$ is fully recursive if all labels from which recursive labels are reachable in $G_{d}$ are mutually recursive.

We can now show:

TheOrem 3.1. : A specialized DTD is strongly recognizable iff it is non-recursive.

Proof: Let $d=\left(\Sigma, \Sigma^{\prime}, d^{\prime}, \mu\right)$ be a specialized DTD. Suppose first that $d$ is strongly recognizable, i.e. $\mathcal{L}(d)$ is regular ${ }^{3}$. Then there exists an FSA $A$ recognizing exactly $\mathcal{L}(d)$. Suppose towards a contradiction that $d^{\prime}$ is recursive and let $a \in \Sigma^{\prime}$ be a recursive label in $d^{\prime}$. Hence there exists a tree $t$ in $S A T\left(d^{\prime}\right)$ where $a$ repeats along one path. The string $[t]$ is of the form $r u_{1} a v_{1} a w \bar{a} v_{2} \bar{a} u_{2} \bar{r}$ where $u_{1} u_{2}$ and $v_{1} v_{2}$ are well-balanced words corresponding to subtrees (or forests) of $t$. By iterating the recursive part of the derivation from $a$ to $a$, we obtain that $[t]_{n}=r u_{1}\left(a v_{1}\right)^{n} a w \bar{a}\left(v_{2} \bar{a}\right)^{n} u_{2} \bar{r}$ is also in $\mathcal{L}\left(d^{\prime}\right)$ for each $n>0$. Thus, all words $\mu\left(\left[t_{n}\right]\right)$ are accepted by the FSA $A$. A simple pumping argument then shows that $\mu\left(r u_{1}\left(a v_{1}\right)^{(n+k)} a w \bar{a}\left(v_{2} \bar{a}\right)^{n} u_{2} \bar{r}\right)$ is also accepted by $A$ for some $k>0$. This is a contradiction, since the string is not well-balanced.

Assume now that $d$ is non-recursive. We can assume wlog that $\Sigma \cap \Sigma^{\prime}=\emptyset$. For each $b \in \Sigma^{\prime}$ construct an FSA $A_{b}$ recognizing $\mu(b) R_{b} \overline{\mu(b)}$, where $R_{b}$ is the regular expression associated to $b$ in $d^{\prime}$. An FSA $A$ recognizing $\mathcal{L}(d)$ is constructed inductively as follows. Let $A_{0}$ be $A_{r}$ where $r$ is the root label. For $i \geq 0, A_{i+1}$ is obtained by modifying $A_{i}$ as follows. For each transition $e=(p, b, q)$ of $A_{i}$, where $b \in \Sigma^{\prime}$ :

1. add a copy $A_{e}$ of $A_{b}$

2. add the transitions $\left(p, \epsilon, i_{e}\right)$ where $i_{e}$ is the start state of $A_{e}$, and $\left(f_{e}, \epsilon, q\right)$ for each accepting state $f_{e}$ of $A_{e}$

3. remove $e$.

Because $d$ is non-recursive this process is sure to terminate. Note that the resulting FSA is over alphabet $\Sigma \cup \bar{\Sigma}$. It is easy to verify that the FSA recognizes $\mathcal{L}(d)$.

To conclude the section, we consider a somewhat surprising converse to Theorem 3.1. One might legitimately wonder if there are type systems other than specialized DTDs that define families $T$ of trees that can be strongly validated by an FSA. Interestingly, the answer turns out to be negative, as shown next.

TheOrem 3.2. : Let $T$ be a set of trees over $\Sigma$. The language $\mathcal{L}(T)$ is regular iff there exists a non-recursive specialized DTD d such that $T=S A T(d)$.

${ }^{3}$ Recall that $\mathcal{L}(d)$ is a language over $\Sigma \cup \bar{\Sigma}$. 
Proof: The "if" part follows from Theorem 3.1. For the "only if" part, suppose $\mathcal{L}(T)$ is regular so is recognized by some FSA $A$. From $A$ we can easily construct a tree-walking automaton $A^{\prime}$ that performs a depth-first traversal of its input, simulating at each step the corresponding move in $A$ and recognizing $T$. Since treewalking automata define regular tree languages, and since specialized DTDs define all regular tree languages (see Preliminaries), there exists a specialized DTD $d$ such that $T=\operatorname{SAT}(d)$. By Theorem 3.1, $d$ is nonrecursive.

\section{VALIDATING WELL-FORMED XML DOCUMENTS}

We now consider the problem of validating an XML document with respect to a given DTD $d$, assuming that the XML document is well formed. As before, we would like to perform the validation using an FSA. The previous requirement that $\mathcal{L}(d)$ be regular is now too strong, because the FSA only needs to work correctly on wellbalanced strings representing trees. The problem can be formalized as follows. Let $\mathcal{L}$ (Tree) denote the language consisting of all string representations of trees over $\Sigma$. The DTD $d$ can be validated by an FSA iff there exists some regular language $R$ such that $\mathcal{L}(d)=\mathcal{L}($ Tree $) \cap R$. Such DTDs are called recognizable. The characterization of recognizable DTDs turns out to be a non-trivial problem. In order to develop some intuition, we start with several examples.

ExAmPLE 4.1. : Let us revisit the DTD $d$ of Example 3.1: $\begin{array}{ll}r \rightarrow a & a \rightarrow a\end{array}$. Recall that $d$ is not strongly recognizable. However, it is recognizable. Indeed, if the input is known to be well balanced, it is sufficient for an FSA to check that the string is of the form $r a^{*} \bar{a}^{*} \bar{r}$. In other words, $\mathcal{L}(d)=\mathcal{L}($ Tree $) \cap r a^{*} \bar{a}^{*} \bar{r}$.

We provide two more examples of recognizable DTDs.

$$
\begin{array}{ll} 
& r \rightarrow a \\
\text { Example 4.2. : Consider the DTD } & a \rightarrow b \\
& b \rightarrow a
\end{array} \text { ? }
$$

with root $r$, which defines trees that are vertical alternations of $a$ and $b$ under root $r$. This DTD can be validated because $\mathcal{L}(d)=\mathcal{L}($ Tree $) \cap r(a b)^{*}(\bar{b}|\bar{a}| \bar{r})^{*}$.

$$
\text { ExAmple 4.3. : Consider the DTD } \begin{aligned}
& a \rightarrow b^{*} \\
& b \rightarrow a^{*}
\end{aligned}
$$

This can be validated by the following FSA that only allows the valid transitions $a b, b a, \bar{a} \bar{b}, \bar{b} \bar{a}, a \bar{a}, b \bar{b}, \bar{a} a, \bar{b} b$ rejecting all the others.

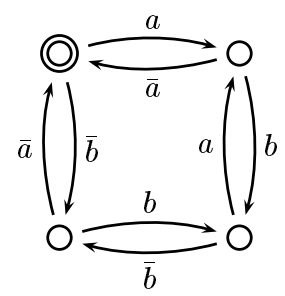

We next provide an example of a DTD that is not recognizable.

$$
\begin{aligned}
\text { EXAMPLE 4.4. : Let } d \text { be the DTD: } & a \rightarrow(a b|c a| \epsilon) \\
& c \rightarrow \epsilon
\end{aligned}
$$

This DTD defines trees of the form:

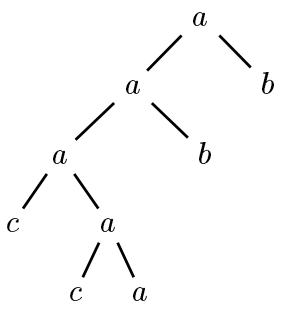

This DTD is not recognizable. Intuitively, even if the document is assumed to be well balanced, an FSA cannot store enough information to recall, when it reads an $\bar{a}$, whether the corresponding node had a left sibling labeled $c$ (in which $b$ is not allowed to its right). The formal proof follows from Lemma 4.2, see Example 4.5.

Also by way of technical warm-up, it is worth noting that conventional wisdom relating to FSA does not necessarily apply when inputs are restricted to well-balanced strings. Basic issues such as equivalence or minimization are quite different in this setting. To illustrate, consider again Example 4.2. The minimal deterministic FSA corresponding to the regular expression $r(a b)^{*}(\bar{b}|\bar{a}| \bar{r})^{*}$ has five states, and it is easily seen that this is minimal among all FSA validating the DTD. However, it is by no means unique - another deterministic FSA with five states equivalent to the first on well-balanced strings but non-isomorphic to it is the minimal one for the regular expression $r(a \mid b)^{*}\left(\bar{b} \bar{a} \bar{r}^{*}\right)^{*}$. Both FSA have the same number of states and agree on the well-balanced strings. However, the two FSA disagree on the non well-balanced words. For instance, the regular expression of Example 4.2 accepts $r a b \bar{b}$ while the one above does not. Thus there is no unique minimal FSA on well-balanced inputs, unlike in the classical setting. In particular, it is not clear how to minimize an FSA validating a given recognizable DTD. However, equivalence of FSA on wellbalanced inputs is decidable in EXPTIME (by a reduction to equivalence of top-down tree automata). It is open whether this can be improved. 
Before proceeding, we make the following useful observation.

LEMma 4.1. : Let $T$ be a set of tree documents over alphabet $\Sigma$. If $\mathcal{L}(T)=\mathcal{L}$ (Tree) $\cap R$ for some regular language $R$, then $T=\operatorname{SAT}(d)$ for some specialized DTD $d$ computable in PTIME from the FSA for $R$.

Proof The construction of $d$ is similar to the classical construction of a CFG for the intersection of another CFG with a regular language, used to show closure of CFL's under intersection with regular languages [10]. The specialized alphabet consists of triples $(p, a, q)$ where $a \in \Sigma$ and $p, q$ are states of the FSA $A_{R}$ for $R$. The specializations of the root $r$ are of the form $\left(q_{0}, r, q_{f}\right)$ where $q_{0}$ is the start state and $q_{f}$ an accepting state of $A_{R}$. The regular language associated to $(p, a, q)$ is $\left\{\left(q_{1}, a_{1}, q_{2}\right)\left(q_{2}, a_{2}, q_{3}\right) \ldots\left(q_{k}, a_{k}, q_{k+1}\right) \mid k>\right.$ $0, a_{1} \ldots a_{k} \in R_{a}, q_{i}$ are states of $A_{R},\left(p, a, q_{1}\right)$ and $\left(q_{k+1}, \bar{a}, q\right)$ are transitions in $\left.A_{R}\right\} \cup\left\{\epsilon \mid \epsilon \in R_{a}\right.$ and $(p, a \bar{a}, q)$ is a transition in $\left.A_{R}\right\}$.

We now attempt to characterize recognizable DTDs. Our basic roadmap is the following. We already know from the previous section that non-recursive DTDs are recognizable, since they are strongly recognizable. We manage to obtain a precise characterization of recognizable DTDs in the case of fully recursive DTDs. The characterization in the general case remains open. However, we make partial progress by providing necessary conditions and then extending them to sufficient conditions for recognizability. Our conjecture is that the necessary conditions we provide are actually also sufficient.

We begin with a first necessary condition in order for a DTD to be recognizable. As will be seen shortly, this condition is not sufficient in general. However, we show in Theorem 4.1 that the condition becomes sufficient in the special case of fully recursive DTDs.

Lemma 4.2. : Let $d$ be a recognizable DTD. Then the following hold, where $\alpha, \beta, u, v, w$ are words over $\Sigma$ while $x, y, z$ (possibly subscripted) are individual symbols:

Let $k$ be a positive integer and $x_{i}, z_{i}, 1 \leq i \leq k$ be mutually recursive symbols of $d$ (not necessarily distinct). If $\alpha x_{1} \beta \in R_{z_{1}}, \alpha^{\prime} x_{k} \beta^{\prime} \in R_{z_{1}}$ and $u_{i} x_{i-1} v_{i} x_{i} w_{i} \in R_{z_{i}}$ for $1<i \leq k$, then $\alpha x_{1} v_{2} x_{2} \cdots v_{k} x_{k} \beta^{\prime}$ must be in $R_{z_{1}}$.

The proof of the lemma relies on a rather involved pumping argument and is sketched below. We first provide some intuition and examples. The condition relates to the inability of an FSA to enforce non-trivial horizontal constraints on the structure of trees when they concern mutually recursive symbols. This stems from the inability to remember the depth of elements, and therefore to determine when nodes are siblings. Very roughly, the rule states that what is allowed at some depth must also be allowed at any depth, modulo limited local constraints that can be enforced. More specifically, if $x_{1}$ and $x_{k}$ are allowed to occur at the same level (under $\left.z_{1}\right)$ and $x_{i-1}$ can be "connected" to $x_{i}$ via $v_{i}$ at some horizontal level for $1<i \leq k$, then $x_{1}$ may be "connected" to $x_{k}$ via the path $x_{1} v_{2} x_{2} \ldots v_{k} x_{k}$ at the same level under $z_{1}$.

\section{Remark:}

Note that the condition above can be formulated as follows for $k=1$. If $x$ and $z$ are mutually recursive, $\alpha x \beta \in R_{z}$ and $\alpha^{\prime} x \beta^{\prime} \in R_{z}$, then $\alpha x \beta^{\prime}$ must also be in $R_{z}$.

We next consider a few examples.

Example 4.5. : Recall the DTD of Example 4.4. It is not recognizable because it does not satisfy the condition in the above lemma for $k=1$. Indeed, $a$ is recursive in the DTD, $R_{a}$ contains $a b$ and $c a$, but it does not contain $c a b$ as required by Lemma 4.2 .

$$
\text { ExAmple 4.6. : Consider the DTD } \begin{aligned}
& a \rightarrow a \mid b \\
& b \rightarrow(a b)^{?}
\end{aligned}
$$

This is not recognizable because it does not satisfy condition of Lemma 4.2 for $k=2$. Indeed, $a$ and $b$ are mutually recursive, $R_{a}$ contains $a$ and $b, R_{b}$ contains $a b$ but $R_{a}$ does not contain $a b$ as required.

Proof of Lemma 4.2 (sketch). Suppose $d$ is validated by an FSA $A$ with $p$ states. For each $a \in \Sigma$ we fix a tree $\hat{a}$ rooted at $a$ and valid wrt $d$. For simplicity, when the context is clear, we also denote by $\hat{a}$ the string $[\hat{a}]$. If $\alpha$ is a word of $a_{1} \cdots a_{m}$ of $\Sigma^{*}, \hat{\alpha}$ denotes the sequence of trees $\hat{a}_{1} \cdots \hat{a}_{m}$.

We will need the following fact, whose proof is a straightforward application of the pumping lemma for regular languages.

FACT 1. : Let $A$ be a deterministic FSA over $\Sigma, u \in$ $\Sigma^{*}$, and $p$ the number of states of $A$. Let $q$ be the state of $A$ reached after reading $u^{k}, k \geq p$ starting from some state $s$. Then the same state $q$ is reached after reading $u^{k+p !}$ starting from state $s$.

We can assume wlog that $z_{1}$ is the root of the documents accepted by $d$.

The proof has two steps. We first construct a tree $T$ in $S A T(d)$, assuming the hypothesis of the condition of the lemma. Then we modify $T$ and obtain another tree $T^{\prime}$ that is also accepted by $A$ and where the pattern required in the conclusion occurs under a node labeled $z_{1}$. The construction of $T$ is somewhat tricky, as we 
have to ensure that a pumping-like argument can be made to show that $T^{\prime}$ is also accepted.

We start by giving some intuition for the construction. Recall that for each $a \in \Sigma, \hat{a}$ denotes a fixed tree rooted at $a$ and valid for $d$, as well as its string representation.

We first define some "pieces" used in the construction of $T$. Since $x_{i}$ and $z_{i}$ are mutually recursive, there is a derivation in $d$ with a path containing $x_{i}$ followed by $z_{i}$ and followed again by $x_{i}$. Let $\hat{x}_{i}^{p !}$ be the tree depicted bellow which consists of $p$ ! iterations of the derivation of $x_{i}$ from $x_{i}$ via $z_{i}$.

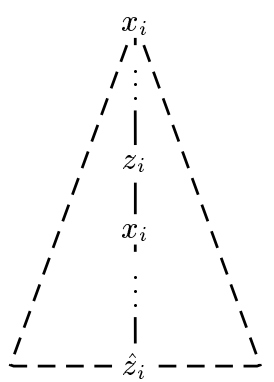

Figure 1: The trees $\hat{x}_{i}^{p !}$

Next, note that each $z_{i}$ can be used to "connect" $\hat{x}_{i}^{p !}$ to $x_{i-1}^{p !}$ by expanding $z_{i}$ into $\hat{u}_{i} x_{i-1} \hat{v}_{i} \hat{x}_{i}^{p !} \hat{w}_{i}$ and further expanding $x_{i-1}$ into $\hat{x}_{i-1}^{p !}$. Also, $x_{1}^{p !}$ can connect to $x_{k}^{p !}$ by expanding $z_{1}$ into $\hat{\alpha}^{\prime} x_{k} \hat{\beta}^{\prime}$. This allows to define by induction the trees $t_{i}$, depicted in Figure 2. Let $T$ be $t_{1}$. Thus, $T$ is obtained by expanding $t_{1}$ with $t_{k}$, which in turn is expanded with $t_{k-1}$, etc. The iteration ends by expanding $t_{2}$ with $x_{1}^{p !}$.

Next, let $T^{\prime}$ be the tree depicted in Figure 3.

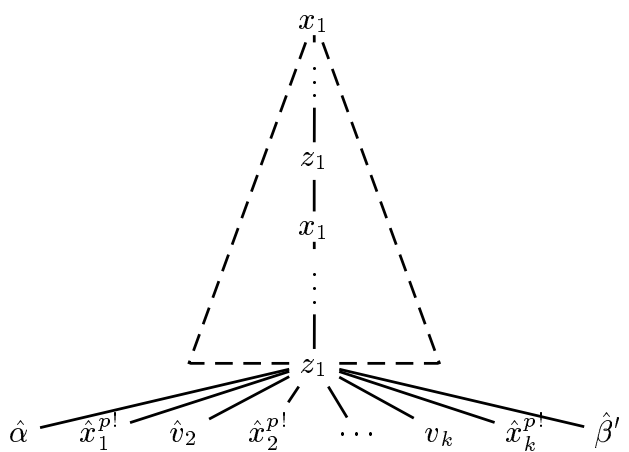

Figure 3: The tree $t^{\prime}$

As we will prove formally, the FSA $A$ (which has $p$ states) cannot distinguish $T$ from $T^{\prime}$. The basic intuition is as follows. Consider the computation of $A$ on $T$ and $T^{\prime}$. The computation can be broken down into two phases: a descending phase from the root consuming all left subtrees along a specified path in each tree, followed by an ascending phase back to the root. In $T$ the path is the one going through the roots of the subtrees $t_{k}$. In $T^{\prime}$ it is the one going through the root of $\hat{x}_{1}^{p !}$. The FSA $A$ reaches the same state after its descending phase in both $T$ and $T^{\prime}$. This is a consequence of Fact 1 , and is shown formally below. For the ascending phase, it is enough to show that $A$ must be in the same state after reading the substrings corresponding to $\hat{x}_{i}^{p !}$ in $T^{\prime}$ and $t_{i}$ in $T$. The argument is inductive. The basis holds because the same state is reached in the descending phase. Suppose next that $A$ is in the same state $q_{i}$ after reading the substrings corresponding to $\hat{x}_{i-1}^{p !}$ in $T^{\prime}$ and $t_{i-1}$ in $T$. Next, $A$ reads $\hat{v}_{i}$ in both trees. This is followed in $T^{\prime}$ by $\hat{x}_{i}^{p !}$, and in $T^{\prime}$ by $\hat{x}_{i}^{p !}$ followed by an additional ascending portion to the root of $t_{i}$. However, the extra ascending string leaves the $A$ in the same state, again as a consequence of Fact 1 . This argument can be iterated to show that $A$ returns to the root of $T$ and $T^{\prime}$ in the same state, so $T$ and $T^{\prime}$ are not distinguished. The formal proof is omitted.

We next show a converse of Lemma 4.2: the necessary condition stated there in order for a DTD to be recognizable is also sufficient when the DTD is fully recursive. To do this, we first show how to construct, from any given DTD $d$, a standard FSA $A_{d}$ that accepts all words in $\mathcal{L}(d)$ (and possibly more). We then show that for fully recursive DTD's $d$ satisfying the conditions of Lemma $4.2, A_{d}$ accepts precisely the words in $\mathcal{L}(d)$.

Although we are primarily interested for the time being in fully recursive DTDs, we provide for later use the construction of $A_{d}$ for arbitrary DTD's.

\section{Construction of the standard FSA}

We now outline the construction of the FSA $A_{d}$. The construction extends the simpler one involved in the proof of Theorem 3.1. Let $d$ be an arbitrary DTD over alphabet $\Sigma$. We will use the dependency graph $G_{d}$ of $d$. Consider the equivalence relation $\equiv$ on $\Sigma$ whose equivalence classes are the strongly connected components of $G_{d}$. Let $\prec$ be the partial order on the classes of $\equiv$ where $A \prec B$ iff for some $a \in A$ and $b \in B$ there is an edge from $a$ to $b$ in $G_{d}$. Note that $\prec$ has a minimum element: the class of the root label. There are generally several maximal elements. We construct $A_{d}$ by induction on $\prec$ starting from the maximal elements.

Let $C$ be a maximal element of $\prec$. This means that for every $c \in C, R_{c}=\{\epsilon\}$ or words in $R_{c}$ contain only symbols that are mutually recursive with $c$. Let $A_{c}$ be an FSA corresponding to the regular expression $R_{c}$. Since $A_{c}$ is non-deterministic, we can assume wlog that $A_{c}$ has no "sink states", i.e. some accepting state is reachable from every state. We can also assume that the sets of states of the FSAs $A_{c}$ are disjoint for different $c$ 's. Let $A_{C}$ be the FSA whose set of states is the union of the sets of states of the FSAs $A_{c}$ for $c \in C$. We do not need to specify at this point initial and final states 

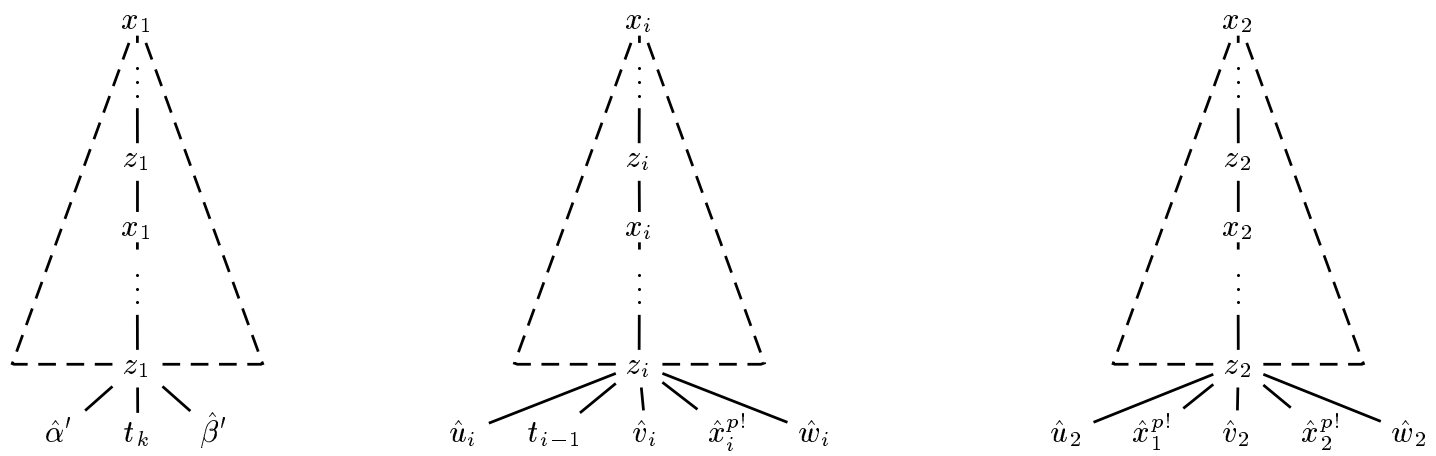

Figure 2: The trees $t_{i}$

for $A_{C}$, but we mark the initial and final states of each of the participating FSAS $A_{c}$ (the initial state for $A_{c}$ is $q_{0}^{c}$ and the final states $\left.f_{1}^{c}, f_{2}^{c}, \ldots\right)$. The transitions are defined as follows. For each transition $\left(q, b, q^{\prime}\right)$ of $A_{c}$ we add to $A_{C}$ the transitions $\left(q, b, q_{0}\right)$ and $\left(f, \bar{b}, q^{\prime}\right)$ for the initial state $q_{0}^{b}$ and for each final state $f_{i}^{b}$ of $A_{b}$.

Now suppose that $C$ is a class of $\equiv$ for which all FSA $A_{D}$ corresponding to classes $D$ such that $C \prec D$ are already constructed. We construct $A_{C}$ as follows. Again, for each $c \in C$, let $A_{c}$ be an FSA corresponding to $R_{c}$ (with disjoint states for distinct $c$ 's). The set of states of $A_{C}$ is the union of the sets of states of the FSAS $A_{c}$ for $c \in$ $C$, similarly for the final states, and the initial state is again left unspecified. The transitions of $A_{C}$ are defined as follows. As in the base case, for each $b \in C$ and transition $\left(q, b, q^{\prime}\right)$ in $A_{c}$ we add to $A_{C}$ the transitions $\left(q, b, q_{0}\right)$ and $\left(f, \bar{b}, q^{\prime}\right)$ for the initial state $q_{0}$ and for each final state $f$ of $A_{b}$. Unlike the base case, we now have to take care of symbols $b$ belonging to some class $B$ for which $C \prec B$. For each such $b$ we add to $A_{C}$ a new disjoint copy of the already constructed $A_{B}$, together with the transitions $\left(q, b, q_{0}\right)$ and $\left(f, \bar{b}, q^{\prime}\right)$ for the copy of the initial state $q_{0}$ and for the copies of each final state $f$ of $A_{b}$.

This induction allows us to construct an FSA $A_{C}$ for the minimum class $C$ containing the root label $r$. The final FSA $A_{d}$ is obtained by adding a new start state $s$ and final state $g$ together with transitions $\left(s, r, q_{0}\right)$ and $(f, \bar{r}, g)$ for the start state $q_{0}$ and each final state $f$ of $A_{r}$.

We illustrate the construction of $A_{d}$ with some examples.

ExAmple 4.7. : Consider the DTD $d \begin{aligned} & r \rightarrow a a \\ & a \rightarrow a\end{aligned}$. The dependency graph $G_{d}$ has the edges $(r, a)$ and $(a, a)$. The classes of $\equiv$ are $\{r\},\{a\}$, and $\{r\} \prec\{a\}$. The FSA $A_{r}$ and $A_{a}$ are :

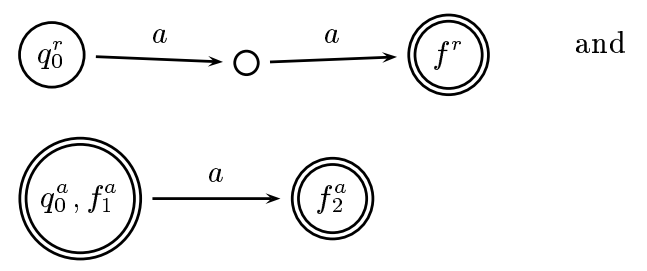

Thus the FSA associated to the equivalence class $\{a\}$ is:

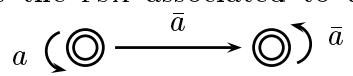

This yields the FSA $A_{d}$ depicted in Figure 4 .

Notice that $A_{d}$ recognizes all the well-balanced words of $\mathcal{L}(d)$. But it also recognizes additional well-balanced

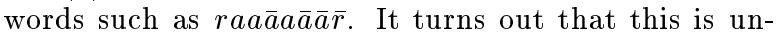
avoidable: there is no automaton that recognizes the above DTD. This will be shown in Lemma 4.4 will show.

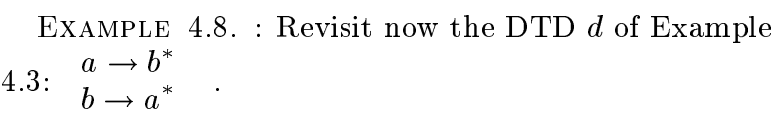

This induces one equivalence class of symbols: $\{a, b\}$. The FSA $A_{a}$ and $A_{b}$ are:

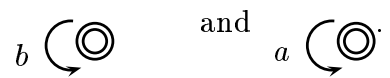

Thus, the FSA associated to the equivalence class $\{a, b\}$ is:

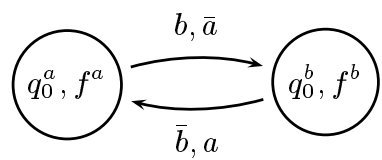

If $a$ is assumed to be the root, this yields the FSA $A_{d}$ :

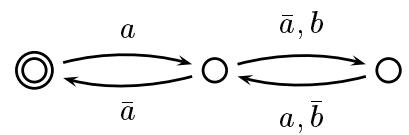

Note that $A_{d}$ is equivalent to the FSA of Example 4.3 


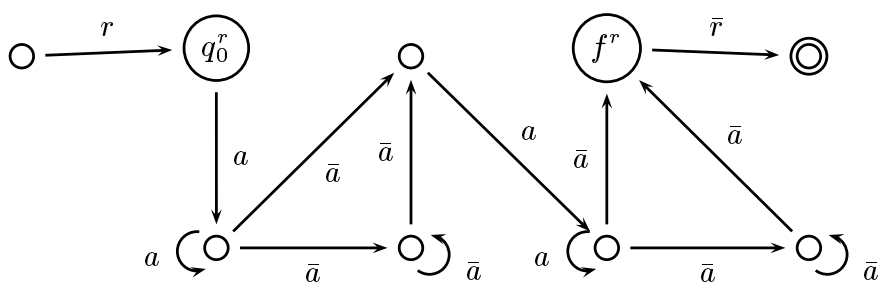

Figure 4: $A_{d}$

and the only well-balanced strings it accepts are those in $\mathcal{L}(d)$.

As expected, we can easily show, by construction, the following property of $A_{d}$.

Lemma 4.3. : For each DTD $d$, let $A_{d}$ be the automaton constructed above. We have:

(i) every word in $\mathcal{L}(d)$ is accepted by $A_{d}$.

(ii) $A_{d}$ can be constructed from $d$ in exponential time.

The construction of $A_{d}$ in the general case takes time $O\left(|d|^{|\prec|}\right)$ where $|d|$ is the maximum size of an FSA for a regular expression of $d$, and $|\prec|$ is the depth of the partial order $\prec$. The exponential is due to the replication of FSA's carried out in the construction.

\section{Remark}

The construction of the FSA $A_{d}$ can be straightforwardly extended to specialized DTDs. Note also that $A_{d}$ is non-deterministic even if the FSA's $A_{b}$ associated to $R_{b}$ are deterministic. The non-determinism stems from the fact that a closing tag $\bar{a}$ may lead to several states.

We can now prove a converse of Lemma 4.2, which yields a precise characterization of recognizable fully recursive DTDs.

THEOREM 4.1. : The following are equivalent for each fully recursive DTD $d$ :

(i) $d$ is recognizable,

(ii) d satisfies the conditions of Lemma 4.2, and

(iii) the set of well-balanced strings accepted by the FSA $A_{d}$ is precisely $\mathcal{L}(d)$.

As a consequence of Theorem 4.1, we can show that it is decidable whether a fully recursive DTD $d$ is recognizable, and therefore whether the standard associated FSA $A_{d}$ can be used to validate it.

THEOREM 4.2. : Given a specialized fully recursive DTD $d$ over fixed alphabet $\Sigma$, it is decidable in EXPTIME whether $d$ is recognizable.
Remark:

The exponential complexity above is due to the construction of deterministic FSA for the regular expressions used by the DTD. If the DTD only uses 1-unambiguous regular expressions, such as required by XML schema [4], the complexity goes down to PTIME.

Let us now consider DTDs that are not fully recursive. Consider again the DTD of Example 4.7. Intuitively, the DTD cannot be recognized because an FSA cannot keep track of the depth in the tree and thus might allow the transition from $\bar{a}$ to $a$ at depth different than 1 . The next lemma formalizes this intuition and provides a second necessary condition for recognizability.

Lemma 4.4. : Let $d$ be a recognizable DTD. Then the following holds, where $\alpha, \beta, u, v, w$ are words over $\Sigma$ while $x, y, z$ (possibly subscripted) are individual symbols:

Let $x_{1}, x_{2}, y, z$ be symbols such that $x_{1}, x_{2}$ and $z$ are mutually recursive in $d$. If $u x_{1} v x_{2} w \in R_{y}$ and $u^{\prime} x_{1} v^{\prime} x_{2} w^{\prime} \in$ $R_{z}$ then $u x_{1} v^{\prime} x_{2} w$ must be in $R_{y}$ and $u^{\prime} x_{1} v x_{2} w^{\prime}$ must be in $R_{z}$.

\section{Remark:}

The condition of Lemma 4.4 can be formulated as follows in the special case when $x_{1}=x_{2}$ : (i) Suppose the occurrences of $x_{1}$ and $x_{2}$ below $z$ are identical. Let $x$ and $z$ be mutually recursive in $d$. If $u x v x w \in R_{y}$ and $u^{\prime} x w^{\prime} \in R_{z}$ then $u^{\prime} x v x w^{\prime} \in R_{z}$ and $u x w \in R_{y}$. (ii) Suppose the occurrences of $x_{1}$ and $x_{2}$ below $y$ are identical. If $u x w \in R_{y}, u^{\prime} x v^{\prime} x w^{\prime} \in R_{z}$ then $u x v^{\prime} x w \in R_{y}$ and $u^{\prime} x w^{\prime} \in R_{z}$.

Example 4.9. : Consider the DTD of Example 4.7 $r \rightarrow a a$
$a \rightarrow a^{?}$ . This DTD is not recognizable because it does not satisfy the condition of Lemma 4.4. Indeed, $a$ is recursive, $R_{r}$ contains $a a, R_{b}$ contains $a$ but $R_{b}$ does not contain $a a$. This violates the condition of the lemma.

$$
\begin{array}{ll} 
& r \rightarrow a b \\
\text { Example 4.10. : Consider the DTD } a \rightarrow b^{*} \\
& b \rightarrow a^{*}
\end{array} \text {. This }
$$

DTD is not recognizable because it does not satisfy the 
condition of Lemma 4.4. Indeed, $a$ and $b$ are mutually recursive, $R_{r}$ contains $a b, R_{b}$ contains $a a$ but $R_{r}$ does not contain $a a b$.

Note that, if we replace the first rule by $r \rightarrow a^{*} b^{*}$, conditions of Lemmas 4.2 and Lemma 4.4 are satisfied and the resulting DTD is recognized by $A_{d}$.

We conjecture that the necessary conditions provided by Lemmas 4.2 and 4.4 are a in fact a precise characterization for DTD recognizability. However, this remains open.

Short of a complete characterization of recognizable DTDs, we provide of characterization of when a DTD $d$ is validated by the standard FSA $A_{d}$. The conditions are those of Lemmas 4.2 and 4.4, together with an additional condition stated next:

(*) Let $\alpha, \beta, u, v, w$ be words over $\Sigma$ and $x, y, z$ (possibly subscripted) be individual symbols. Let $k$ and $k^{\prime}$ be positive integers. Let $\left(x_{i}\right)_{1 \leq i \leq k},\left(z_{i}\right)_{2 \leq i \leq k}$, $\left(x_{i}^{\prime}\right)_{1 \leq i \leq k^{\prime}},\left(z_{i}^{\prime}\right)_{2 \leq i \leq k^{\prime}}$, and $y$ be symbols of $\Sigma$ such that $x_{1}=x_{1}^{\prime}, x_{k}=x_{k^{\prime}}^{\prime}$, and all the $x_{i}, x_{i}^{\prime}, z_{i}, z_{i}^{\prime}$ are mutually recursive in $d$ (not necessarily distinct). If $u x_{1} v_{1} x_{2} \cdots v_{k-1} x_{k} w \in R_{y}$ and for each $2 \leq i \leq k$ we have $\left\{\alpha_{i} x_{i-1} \beta_{i}, \alpha_{i}^{\prime} x_{i} \beta_{i}^{\prime}\right\} \subset R_{z_{i}}$ and for each $2 \leq i \leq k$ we have $u_{i}^{\prime} x_{i-1} v_{i}^{\prime} x_{i} w_{i}^{\prime} \in R_{z_{i}^{\prime}}$ then $u x_{1}^{\prime} v_{1}^{\prime} \cdots x_{k}^{\prime} w$ must be in $R_{y}$ and, for each $2 \leq i \leq k, \alpha_{i} x_{i-1} v_{i-1} x_{i} \beta^{\prime}$ must be in $R_{z_{i}}$.

The next result provides a precise characterization of the DTDs $d$ that are validated by the standard FSA $A_{d}$.

Theorem 4.3. : Let $d$ be a DTD. The following are equivalent:

(i) d satisfies (*) and the conditions of Lemmas 4.2 and 4.4 , and

(ii) the set of well-balanced strings accepted by the FSA $A_{d}$ is precisely $\mathcal{L}(d)$.

We note that the conditions of Theorem 4.3 can be verified in time doubly exponential with respect to $d$. This is done by checking directly that $A_{d}$ validates $d$, as follows. We first build a specialized DTD $d^{\prime}$ such that $S A T\left(d^{\prime}\right)$ consists of the trees accepted by $A_{d}$. This can be done in Exptime by Lemma 4.1. Next, the equivalence of $d$ and $d^{\prime}$ can be checked in Exptime using a tree automata equivalence test.

To understand why the conditions in Theorem 4.3 are not a complete characterization of recognizable DTDs, consider the following example, that provides a recognizable DTD $d$ violating $(*)$. For this DTD, we will exhibit an FSA different from the standard $A_{d}$, that validates it. $r \rightarrow a \alpha b \beta c$

$a \rightarrow a d \mid \epsilon$

ExAmple 4.11. : Consider the DTD $d: d \rightarrow d c \mid \epsilon$

$b \rightarrow a|b| \epsilon$

$c \rightarrow b|c| \epsilon$

First notice that $A_{d}$ does not recognize this DTD because $d$ violates $\left(^{*}\right)$. Indeed the DTD satisfies the premise of $\left(^{*}\right)$ but not its conclusion. For example, adc is not in $R_{r}$ as required. However, consider the FSA that works like $A_{d}$, but additionally counts the number of transitions $\bar{a} d$ and $\bar{d} \bar{a}$ modulo 2 and accepts only if the two are equal. It can be verified that this FSA validates $d$.

In summary, the conditions of Lemmas 4.2 and 4.4 are necessary in order for a DTD to be recognizable. The conditions of Theorem 4.3 are sufficient, and in particular provide a precise characterization of when the standard FSA works. The complete characterization of recognizable DTDs remains open.

\section{ALTERNATIVE APPROACHES TO VALIDATION}

We next consider two alternative approaches for validating DTDs that are not recognizable. The first is to relax the constant memory requirement. The second consists in refining the original DTD by adding information allowing it to be validated by an FSA.

\section{Validation with bounded stack}

We begin with relaxing the memory requirement. Specifically, we allow as auxiliary memory a stack whose depth is bounded in the depth of the XML document. The requirement that validation be done in a single, deterministic pass is maintained. This approach is appealing in practice, because many XML documents tend to be shallow even if their DTDs are recursive. We start with a simple example.

ExAmple 5.1. : Consider the DTD of Example 4.7 $r \rightarrow a a$
$a \rightarrow a^{?}$$\quad$ which is not recognizable. However, a de$a \rightarrow a$
terministic PDA can validate the DTD by allowing only transitions $a a$ and $\bar{a} \bar{a}$ and remembering the current depth using the stack. In addition, the PDA allows a single transition $\bar{a} a$ and only at depth one. Note that this PDA is deterministic and its stack never exceeds the depth of the tree represented by the well-balanced input string.

Rather surprisingly, we can show that every specialized DTD can be strongly validated by a deterministic PDA. When the input string is well-balanced, the stack of the PDA is bounded in the depth of the tree represented by the input string.

TheOrem 5.1. : Let $d$ be a specialized DTD. There exists a deterministic PDA that accepts precisely $\mathcal{L}(d)$ 
using a stack of depth bounded by the maximum number of unmatched open tags occurring as the input is read from left to right. In particular, if the input string is well-balanced, the depth of the stack is bounded by the depth of the tree represented by the input string.

Proof: Let $d=\left(\Sigma, \Sigma^{\prime}, d^{\prime}, \mu\right)$. Recall that $d^{\prime}$ is a DTD over $\Sigma^{\prime}$ and $\mu$ is the associated specialization mapping. We wish to check whether a string $w$ over $\Sigma \cup \bar{\Sigma}$ represents a tree satisfying $d$. The stack is used to check that the string represents a tree and to keep information about the path from the root to the currently visited node in the tree. For each node along the path, the stack keeps a set of candidate specializations for the node label, compatible with the information seen so far. Intuitively, a candidate specialization $a$ is acceptable if there are acceptable specializations of its children whose sequence forms a word in the regular language $R_{a}$ associated to $a$ by $d^{\prime}$. The PDA must verify this recursively, and accept the input if the root is left with at least one acceptable specialization. To achieve this, the PDA simulates the run of the FSA for $R_{a}$ on the children of a given node with candidate specialization $a$. This is done by keeping on the stack, together with each such $a$, the set of states reached in the FSA for $R_{a}$ after reading the sequence of children seen so far, with their respective allowed specializations. This can be done because the stack symbol containing this information for a given node becomes the top of the stack every time one of its subtrees has been completely read. After reading the entire sequence of its children with their allowed specializations, a candidate specialization $a$ for the node is discounted unless the associated set of states contains some accept state in the FSA for $R_{a}$.

We now describe the PDA in more detail. For each $a \in \Sigma^{\prime}$ let $A_{a}$ be the standard non-deterministic FSA for $R_{a}$, with start state $q_{a}^{0}$. Let $Q$ be the disjoint union of the sets of states of the FSA's $A_{a}$. The stack alphabet of the PDA, denoted $V$, consists of symbols of the form $(a, S)$ where $a \in \Sigma$, and $S$ is a set of elements $\left\langle a^{\prime}, H\right\rangle$ such that $a^{\prime} \in \Sigma^{\prime}, \mu\left(a^{\prime}\right)=a$, and $H$ is a subset of the states $Q$. Thus, $V$ is a subset of $\Sigma \times 2^{\Sigma^{\prime} \times 2^{Q}}$. The transitions work as follows. When $a \in \Sigma$ is read, the symbol $\left(a,\left\{\left\langle a^{\prime},\left\{q_{a^{\prime}}^{0}\right\}\right\rangle \mid a^{\prime} \in \Sigma^{\prime}, a=\mu\left(a^{\prime}\right)\right\}\right)$ of $V$ is pushed on the stack. When a symbol $\bar{a} \in \bar{\Sigma}$ is read, the PDA pops the current stack symbol. If the input string is well balanced, the top of the stack must be of the form $(a, S)$; otherwise the input is rejected. Note that, since the subtree rooted at $a$ has been completely processed, we now know which of the candidate specializations of $a$ are acceptable: they are the $a^{\prime}$ such that $\left\langle a^{\prime}, H\right\rangle \in S$ and $H$ contains some accepting state of $A_{a^{\prime}}$. At this point the new top of the stack symbol, say $(b, T)$, needs to be updated. The symbol is popped and replaced at the top of the stack by $(b$, new $(T))$ where new $(T)$ contains, for each $\left\langle b^{\prime}, B^{\prime}\right\rangle \in T$ the pair $\left\langle b^{\prime}\right.$, new $\left.\left(B^{\prime}\right)\right\rangle$ where new $\left(B^{\prime}\right)$ contains the states $q^{\prime}$ such that $\left(q, a^{\prime}, q^{\prime}\right)$ is a transition of the FSA $A_{b^{\prime}}$ for some $q \in B^{\prime}$ and some allowed specialization $a^{\prime}$ of $a$ occurring in $S$. Finally, the
PDA accepts if the root node labeled $r$ has at least one acceptable specialization $r^{\prime}$. This information is available in the last symbol popped from the stack before it becomes empty.

It is straightforward to check that the above PDA accepts $\mathcal{L}(d)$.

\section{Refining the DTD}

We finally consider an approach to validation orthogonal to the ones examined so far. It consists of refining the given DTD by providing in the tags additional information that can be used for validation. The refinement is formalized by a specialization of the original DTD. More precisely, we can show the following.

TheOREM 5.2. : For every DTD d over $\Sigma$ there exists an equivalent specialized $D T D \bar{d}=\left(\Sigma, \Sigma^{\prime}, d^{\prime}, \mu\right)$ of size quadratic in $d$ such that $d^{\prime}$ is recognizable.

Proof: For each $a \in \Sigma$, let $A_{a}$ be a standard nondeterministic FSA for the regular language $R_{a}$ specified for $a$ by the DTD $d$. The idea for constructing the specialized DTD $\bar{d}$ is straightforward: keep track in the tags of the children of a node $a$ of the state of $A_{a}$ in an accepting computation on the sequence of children tags. More precisely, let $Q$ be the disjoint union of the sets of states of the FSA's $A_{a}$ and let $\Sigma^{\prime}=$ $\Sigma \times Q$. The DTD $d^{\prime}$ associates to each symbol $(a, q)$ in $\Sigma^{\prime}$ the regular language consisting of all words of the form $\left(a_{1}, q_{1}\right)\left(a_{2}, q_{2}\right) \ldots\left(a_{k}, q_{k}\right)$ such that $a_{1} a_{2} \ldots a_{k} \in$ $R_{a}$ and $\left(q_{i-1}, a_{i}, q_{i}\right)$ are valid transitions in $A_{a}, 1 \leq i \leq$ $k$, where $q_{0}$ is the start state and $q_{k}$ an accept state for $A_{a}$. Clearly, the specialized DTD $\bar{d}$ is equivalent to $d$. An FSA can validate well-balanced input strings wrt the DTD $d^{\prime}$ by allowing only the following transitions:

1. $(a, q)\left(a_{1}, q_{1}\right)$ where $\left(q_{0}, a_{1}, q_{1}\right)$ is a transition in $A_{a}$ and $q_{0}$ is the start state of $A_{a}$;

2. $\overline{(a, q)}(b, p)$ where $(q, b, p)$ is a transition in the $\mathrm{FSA}$ to which $q$ belongs.

3. $\overline{(a, q)} \overline{(b, p)}$ where $q$ is an accepting state in the FSA to which it belongs.

Example 5.2. : Revisit the DTD of Example 4.7 $r \rightarrow a a$
$a \rightarrow a$$\quad$ which is not recognizable. However the fol-

$\begin{array}{ll} & r \rightarrow a_{1} a_{2} \\ \text { lowing DTD } & a_{1} \rightarrow a_{1}^{?} \\ a_{2} \rightarrow a_{2}^{?}\end{array}$ is recognizable (by the regular expression $\left.r a_{1}^{*} \bar{a}_{1}^{*} a_{2}^{*} \bar{a}_{2}^{*} \bar{r}\right)$ and defines a similar family of tree documents.

\section{CONCLUSIONS}

This paper provides a first step towards the formal investigation of processing streaming XML. We focused 
on the problem of on-line validation of streaming XML documents with respect to a DTD, under memory constraints. The main results provide conditions under which validation can be done in a single pass and constant memory, using an FSA. We also considered alternative approaches by relaxing the constant memory requirement or by enriching the DTD with additional information that can be used in validation.

Several questions remain open. Mainly, a precise characterization of recognizable DTDs is not yet available, except in the fully recursive case. For the general case, we conjecture that (i) the necessary conditions we provided for a DTD to be recognizable are also sufficient, and (ii) whenever a DTD $d$ is recognizable it can be validated by the standard FSA $A_{d}$ augmented with counting certain patterns modulo 2, as discussed in Example 4.11 .

Another interesting open problem concerns characterizing the specialized DTDs that are recognizable. It can be seen that the conditions we provided for recognizable DTDs no longer work when specialization is allowed. Indeed, the problem seems considerably harder in this case. Note that, since every recognizable family of trees is necessarily definable by a specialized DTD (Lemma 4.1 ), characterizing the recognizable specialized DTDs would essentially close the problem of understanding which families of trees can be validated by FSA.

Finally, it would be useful to exhibit natural classes of DTDs that can always be validated by an FSA, by providing restricted specification languages for document structure that are powerful enough for a wide range of applications of practical interest.

Beyond the immediate focus on validation, we expect that the techniques developed here will also be useful in investigating the more complex problem of querying streaming XML documents.

\section{Acknowledgment}

We wish to thank Bertram Ludaescher and Yannis Papakonstantinou for pointing to us the problem of processing streaming XML. We are also grateful to Serge Abiteboul and Tova Milo for interesting discussions on the topic.

\section{REFERENCES}

[1] A.V. Aho and J.D. Ullman. Translations on a context free grammar. Information and Control, 19(19):439-475, 1971.

[2] C. Beeri and T. Milo. Schemas for integration and translation of structured and semi-structured data. In Int'l. Conf. on Database Theory, pages 296-313, 1999.

[3] S. Babu and J. Widom. Continuous Queries over Data Streams. In Sigmod Record, Sept 2001.
[4] A. Bruggemann-Klein and D. Wood. One-unambiguous regular languages. Information and Computation, 142(2):182-206, May 1998.

[5] A. Bruggemann-Klein, M. Murata, and D. Wood. Regular tree and regular hedge languages over non-ranked alphabets. Hong Kong Univ. of Science and Technology Computer Science Center Research Report HKUST-TCSC-2001-05, 2001. Available at http://www.cs.ust.hk/tcsc/RR/2001-05.ps.gz.

[6] J. Chen et al. NiagaraCQ: A Scalable Continuous Query System for Internet Databases. In Proc. ACM SIGMOD Conf., Dallas, TX, June 2000.

[7] S. Cluet, C. Delobel, J. Simeon, and K. Smaga. Your mediators need data conversion! In Proc. ACM SIGMOD Conf., pages 177-188, 1998.

[8] J. Engelfriet and H. J. Hoogeboom. Tree-walking pebble automata. In J. Karhum ki, H. Maurer, G. Paun and G. Rozenberg, eds., Jewels are forever, contributions to Theoretical Computer Science in honor of Arto Salomaa, pp. 72-83, Springer-Verlag, 1999.

[9] J. Engelfriet, H. J. Hoogeboom and J-P. van Best. Trips on trees. Acta Cybernetica, 14, pp. 51-64, 1999.

[10] S. Ginsburg. The Mathematical Theory of Context-Free Languages. McGraw-Hill, 1966.

[11] J. E. Hopcroft and J. D. Ullman. Introduction to Automata Theory, Languages, and Computation. Addison-Wesley, 1979.

[12] Z. Ives, Alon Levy and D. Weld. Efficient Evaluation of Regular Path Expressions on Streaming XML Data. Technical Report, University of Washington, 2000.

[13] Z. Ives, Alon Levy and D. Weld. Integrating Network-Bound XML Data. Data Engineering Bulletin, 24(2), 2001.

[14] Ling Liu and Calton Pu and Wei Tang and Wei Han. Conquer: A continual query system for update monitoring in the WWW. In International Journal of Computer Systems, Science and Engineering, 2000.

[15] F. Neven and T. Schwentick. On the Power of Tree-Walking Automata. ICALP 2000: 547-560.

[16] Benjamin Nguyen, Serge Abiteboul, Grégory Cobena, Mihai Preda. Monitoring XML data on the Web. In Proc. ACM SIGMOD Conf., pp.437-448, 2001.

[17] Y. Papakonstantinou and V. Vianu. DTD inference for views of XML data. In Proc. $A C M$ PODS, pp. 35-46, 2000.

[18] G.Rozenberg and A. Salomaa. Handbook of Formal Language, vol. 3. Springer Verlag, 1997. 